heblichen Mengen, tritt nach mehrtägiger Einwirkung eine Gerbung ein. Dabei verfärbt sich die Lösung und auch die Haut sehr stark wie es ja auch schon G e r $\mathbf{n g}$ r o $B$ bei dem Protocatechualdehyd unter Zusatz von Soda oder in der damit gegerbten Haut festgestellt hat. Bei dem Resorcyl-Aldehyd tritt aber eine viel intensivere Dunkelfärbung ein, die auf die Bïldung hochkolloider Systeme zurückzuführen ist, in ähnlicher Weise, wie sie auch schon bei Chinongerbung auch schon ohne Sodazusatz, auftritt. Die Gerbung rührt demnach nicht von der Aldehydgruppe her und auch von keiner chemischen Wechselwirkung der Haut selbst oder den Bestandteilen der Haut. Es ist demnach ganz richtig, wie Ge r n g r o B sagt, daß nicht di Aldehydgruppen bei diesen Polyphenolen, sondern die Hydroxylgruppen für die Gerbung verantwortlich zu machen sind und zwar nach meiner Theorie gerade die in $\mathrm{Orthostellung}$ be find li che Hydroxylgruppe, die über die Bildung von Chinon schließlich zu kolloiden huminartigen Verbindungen führt, und erst dann tritt der Gerbeffekt ein. Die Aldehydgruppe ist also in diesem Falle für die Gerbung ganz nebensächlich, und wenn auch nebenhe mit den Spaltprodukten oder Aminosäuren der Haut eine Wechselwirkung zu Methylenaminosäuren eintritt, so sind diese wie bei allen anderen derartigen Kondensationsverbindungen wasserlöslicher $\mathrm{Na}$ tur, die also zur Hervorbringung des Gerbeffektes, der auf die Wasserunlöslichkeit hinausläuft, gar nicht mit in Betracht gezogen werden können.

Auf Grund dieser chemischen Vorgänge und Veränderungen de o-Protocatechualdehydlösung in alkalischer Lösung sind auch die Adsorptionsversuche, die $\mathbf{G}$ e $\mathbf{r} \mathbf{n} \mathrm{g}$ r o $\mathbf{B}$ noch weiter anführt, für den Gerbvorgang selbst gar nicht zu berücksichtigen, denn der gerbende Körper in dem Protocatechualdehyd ist ja letzten Endes nicht de Aldehyd selbst, sondern die infolge chemischer Veränderungen daraus entstehenden Verbindungen. Die sämtlichen von $\mathbf{G}$ e $\mathbf{r} \mathbf{n}$ g r o $B$ am Schlusse in der Zusammenstellung niedergelegten Fest stellungen über den Nachweis von reaktionsfähigen basischen Grup pen im Kollagen einerseits und das Vorliegen von chemischen Reak tionen beim Gerbprozeß andererseits können als widerlegt und nich stichhaltig betrachtet werden. Diese oder ähnliche Versuche wären nur dann von Bedeutung, wenn der Nachweis dafür vorläge, daß der Eiweißkörper, in diesem Falle die Hautsubstanz, ein einheitliches, chemisches Individuum wären. Das ist aber bisher in keinem Fall gelunge ${ }^{38}$ ), und auch Gerngro $B$ hat diesen Beweis nicht ange treten. Er geht also bei der Erklärung dieser Versuche von einer Hypothese aus, die unrichtig ist, denn man kann sich sehr leicht von dem Gegenteil überzeugen, daß das Hautpulver aus einem Gemisch aller möglichen Einzelbestandteile der Eiweißkörper besteht, die in adsorbiertem Zustande festgehalten werden. Wahrscheinlich ist das Adsorptionsvermögen des unveränderten Eiweißkörpers im vitalen Proze $\hat{B}$ gegenüber freien Aminosäuren und anderen Säuren sehr groß. Erst neuerdings hat A bderh a ld e $n^{19}$ ) festgestellt, daß Amino säuren gegenüber kolloiden Stoffen ein nicht unerhebliches Adsorp tionsvermögen besitzen. Entsteht also aus dem Kollagen unter der Annahme, daß es im lebenden Körper aus einem einheitlichen Indi viduum besteht, irgend ein Abbauprodukt oder eine Aminosänre so wird diese natürlich zunächst so viel durch Adsorption festge halten oder nur so viel Anteile davon an Wasser oder andere Flüssig keiten abgegeben, als an Überschuß oder zur Herstellung des Gleichgewichtes vorhanden ist. Mit der Untersuchung der GesetzmäBig. keiten der von der Haut an die adsorbierte Lösung abgegebenen Substanzmengen habe ich mich ganz kürzlich in umfangreichen Arbeiten ${ }^{20}$ ) beschäftigt, und dadurch werden alle diejenigen Theorien, welche keine Rücksicht auf die eigentümlichen Zustandsverhältnisse der Haut nehmen, vollständig hinfällig und damit auch die Anschau ung von $\mathrm{G}$ e r $\mathbf{n} \mathbf{g} \mathbf{r}$ o $\mathrm{B}$. In noch weiteren kommenden Arbeiten werde ich auch den Nachweis liefern, daß der Verlauf der Gerbvorgänge ein gainz anderer ist, wie etwa bei den gewöhnlichen Ad sorptionsvorgängen, wie man sie bei den Eiweißverbindungen gegenüber Säuren beobachtet.

Nach meiner Theorie lautet die Definition für die Ledersubstanz folgendermaßen

Leder ist tierische Haut, deren Elementar teilchen in Form von submikroskopischen Misch. und Schichtkryställchen gegenüber hydroly tischen. und fermentativen Einflüssen ge sch ütztsind.

Ich werde demnächst Gelegenheit nehmen, an dieser Stelle noch einmal auf die Theorie der Gerbung zurückzukommen. [A. 84.]

18) Vgl. S c hulz, Allgem. Chemie der Eiweißkörper. Stutt. gart 1907. S. $284,295,346,347,358$.

19) Fermentforschung II, Leipzig 1919. S. 74-225.

20) M o e I l e r, Die Beziehungen zwischen Hydrolyse und Adsorption, Collegium 1920, Nr. 599 u. ff.; Ledertechn. Rundschau Nr. $12-141920$.

\section{Die fossilen Brennstoffe und ihre Verwertung im Jahre 1919.}

Von Dr. A. FürTh

(Schlub von Selte 220.)

Gewinnung, Behandlung und Verwertung der Ne ben produkte.

A m m on a k. In einem Vortrag auf der 24. Hauptversammlung der Deutschen Bunsengesellschaft macht C. B o s c h (Z. f. Elcktrochem. 24, 361-369) interessante Mitteilungen über die Verarbeitung ron Ammoniak auf Düngesalze. Er geht hierbei auch auf die Herstellung von Ammonsulfat aus Gaswasser, Gips und Kohlensäure, ferner aus Ammoniak und schwefliger Säure ein. Auch Superphosphat eignet sich zur Absorption von Ammoniak und gibt ein gutes Düngemittel. Ammonchlorid wird ebenfalls zu Düngczwecken dargestellt. Die Produkte der Ammoniakverbrennung werden zu Natrium-, Ammonium und Calciumnitrat verarbeitet. letzteres wird in ein völlig lufttrockenes Pulver übergeführt, indem die $4 \mathrm{Mol}$. Krystallwasser durch Harnstoff ersetzt werden. Harnstoff, der auch aus Ammoniak und zwar aus einer Mischung von Ammoncarbonat mit -carbaminat durch Erhitzen hergestellt wird, ist an sich auch ein gutes Düngemittel für den'Tabakbau. - Zur Entfernung von freier Säure aus dem Ammonsulfat schlägt J. T. S h e a r d (Chem. News 118, 232--233) vor, die Krystalle einem fein verteilten Wassernebel auszusetzen, durch den die anhaftende saure Mutterlauge genügend aber immerhin weniger als beim direkten Waschen, verdinnt wird. - H e i n e k e n (J. f. Gasbel. 62, 30-31) beschreibt eine einfache, für kleine Gaswerke bestimmte Salzgewinnungsanlage, die mit Abfallsalzsäure oder Bisulfat und mit direkter Eindampfung arbeitet. - Eine für die Abtreibung des Ammoniaks aus Gaswrsse geeignete Kolonne hat sich die $\mathrm{Gew}$ e $r$ k o h a $\mathrm{t}$ des $\mathrm{S}$ t e in kohlenbergwerks Lothringen (D. R. P. 312933) schützen lassen: sie besteht aus übereinander angeordneten $\mathrm{Ab}$ teilen, in denen die Gase zwangläufig durch Schleier der herab rieselnden Flüssigkeit geführt werden. Das Charakteristische ist hierbei, daß die Ringe mit zwei Arten von Tauchungen ausgerüstet sind.

Von den Verfahren zur Darstellung von Ammonsulfat aus Gips und Gaswasser wäre vor allem das von C. Ot to \& Co. (D. R. P. 299 622) zu erwähnen, das dadureh gekennzeiohnet ist, daß das bei der Reaktion nicht umgesetzte Ammoniak des Gaswassers aus der entstchenden Sulfatlauge durch warmes gerrinigtes Destillationsgas ausgetrieben und in den Gasstrom der Destillationsanlage zur Auf nahime weiterer Kohlensäure zurückgeleitet wird. - Die $\mathrm{Ch}$ e mische Industrie-Aktien-Gesellsebaft und F. W o lf (D. R. P. 299 752) bringen die Kohlensäure unter Drucl: auf das Gips-Gaswassergemisch zur Finwirkung, um die Umsetzung da durch zu beschleunigen. - Wesentlich ist eine $\mathrm{MaBregel}$, die die Badische Anilin - und Sod a f a b r ik (D. R. P. 300724 ) bei diesem Verfahren trifft: Der Gips wird bei $300^{\circ}$ gebrannt, wodurch der resultierende Kalkschlamm sich besser filtrieren läßt. Nach D. R. P. 299621 , welches eine Reaktionskolonne zur Umsetzung ron Ammoncarbonat mit Gips zu Ammonsulfat betrifft, setzen C. Utt o \& Co. der Gipsaufschlämmung indifferente Stoffe we z. B. Kochsalz zu, um die Lösungsfähigkeit des Gipses zu erhöhen, und wählen die Konzentration der Gipsaufschlämmung so, daß ein großer Teil des entstandenen Ammonsulfats in fester Form im Schlamm bleibt. Den Ammoniak- und Kohlensäuredämpfen wird so viel gespannter Wasserdampf zugemischt, daß die ent stehenden Laugen ammoniakfrei sind, und eine günstige Temperaturerhöhung im Apparat erzielt wird. - Das D. R. P. 309975 betrifft eine Verbesserung der Kolonne derselben Firma, bei der der dreh. bare innere Teil, welcher Tassen, Dampfhauben oder Schaber trägt, aus einem Hohlzylinder besteht, der aus einzelnen Ringen zusammengesetz't ist. - Zur Geschichte der Ammonsulfatgewinnung mit Hilfe von Gips stellt übrigens A. S a n d e r (Chem.-Ztg. 43, 661) fest, daß schon vor 100 Jahren Ammonsulfat in der $K$. Salmiakfabrik Nußdorf als Zwischenprodukt bei der Herstellung von Salmiak aus Ammoncarbonat und Gips gewonnen wurde. - Da nach dem D. R.P. 315622 der Badis chen Anilin - und Sodaf a brik aus Ammonsulfat durch Erhitzen mit heißen Flammengasen Ammonbisulfat erzeugt wird, welches in vielen Fällen statt freier Schwefelsäure verwendet werden kann, so erscheint auch damit ein Weg gegeben, der Schwefelsäurenot zu steuern. - Als Ersatz für Ammonsulfat wird neuerdings Ammoncarbonat empfohlen, das aber für Düngezwecke bisher wegen seiner Hygroskopizität und Unheständig keit nicht geeignet war. Nach einem Verfahren des $O$ s terr. Vereines für chemische und metallurgische Produ kti on (D. R. P. 313 827), das in einer Nachbehand]ung des krystallinjsch gefällten Bicurbonats mit Kohlensäure unter Druck (2-3 Atm.) und Brikettieren vor oder nach der Kohlensäurebehandlung besteht, wird das Salz in geruchloser, beständiger Form erhalten.

Die Gewerkschaft des Steinkoh len bergwerks L o t h r i n g e n (D. R. P. 313928 ) hat sich ein Verfahren zur kon. 
tinuierlichen Entsäuerung des Gaswassers schützen lassen, nach welchem das durch teilweise Entsäuerung entstehende Gasgemisch durch Waschen von Ammoniak befreit und als reines Schwefolwasserstoff-Kohlensäuregemisch abgeführt, das hauptsächlich ammoniakhaltige Waschwasser aber in den Entsäurer zurückgeführt wird. Das aus der Waschkolonne austretende Reinammoniak wird in hochkonzentrierte Ammoniaklösung übergeführt, die zum Waschen der geküblten Dämpfe benutzt wird, so daß die Waschlaug^ nur Schwefelwasserstoff und Kohlensäure enthält, wobei die schwefelwasserstoffreichere Lauge im oberen Teil, die kohlensäurereichere im unteren Teil der Kolonne abgezogen wird. - A. M a r s d e n (J. Soc. Chem. Ind. 37, T. 230-232) erörtert die Verlustursachen bei der Herstellung von konzentrierter Ammoniakf]üssigkeit, die zurückzuführen sind auf eine neue Verarbeitungsart des Gaswassers, die seit dem Kriege geübt wird. - Nach A. T h a u (Glückauf 5.5, 241-243) lassen sich im Ammoniakgewinnungsbetriebe erhebliche Bleiersparnisse erreichen, wenn die Sïttiger mit säurefesten Zementplatten ausgemauert werden, wenn man die Laugeabtropfbehälter aus Beton herstellt, und wenn für die Ejektoren, Abtropfpfannen, Säureleitungen andere widerstandsfähige Metalle verwendet werden.

Bisher war man der Ansicht, daß das dem Salz die bläuliche Färbung verleihende Berlinerblau im Sättiger durch Reaktion.auf das in der Schwefelsäure enthaltene Eisen entsteht. A. T h a u (Glückauf 55, 42-44) widerlegt diese Anschauung und führt die Bildung auf eine vorangegangene Schwefeleisenentstehung in der Oberkolonne des Abtreibers und der Ammoniakdampfleitung zuräck. - Die Möglichkeit der Ammoniakgewinnung in Teerdestillations. betrieben bespricht C. R. W o od ward (Chem. Met. Eng. 18, 299-301). Da das durch Destillation erhaltene Ammoniak zu 99\% als freies Ammoniak vorhanden ist, so erübrigt sich die Verwendung von Kalk bei der Konzentration. - Eine Methode zur Reinigung der Ammoniakabwässer gibt W. D o m i n i k (Metan 2, 13-17) an.

Benzol. T. P. A. (Umschau 23, 312-314) beschreibt die Benzolgewinnung aus Kohlendestillationsgasen an Hand von $\mathrm{Ab}$ bildungen. - Ửber die diesbezüglichen Verhältnisse in den Gaswerken von Paris und Umgebung informiert ein Aufsatz von $L$. Lind e t (Bll. Soc. encour. ind. nat. 131, 133-137). - Eine Vorrichtung zur fraktionierten Destillation von gesättigtem Waschöl der Benzolfabrikation ohne Anwendung von Dampf hat sich C. W e i r a u c h (D. R. P. 316 653) schützen lassen: als Heizmittel kommt ein Gemisch von Luft und rauchfreien Feuergasen zur Verwendung, das in parallelen Abzweigungen alle Abteilungen durchströmt, während sich über dem Heizraum jeder Abteilung ein Kolonnenaufsatz. erhebt, aus dem das Rückflußkondensat der folgenden Abteilung zufließt. A. B a r i l (Chimie et Industrie 2, 1013-1016) und C h. B e r t h e lot (Chimie et Industrie 2, 1017-1023) beschreiben die Gewinnung und Rektifizierung des Benzols und die hierbei verwendeten Apparate. - Über die Benzolgewinnung aus italienischen Erzeugnissen, und zwar einerseits aus Leuchtgas, andererseits aus einem an sich nicht verwertbaren Rohpofroleum von Ripi, Provinz Rom, berichtet A.G.R od a n o (Annali Chim. Appl. 8, 7-11 u. 10, 23-26). - Ein Verfahren zur Gewinnung von leichten Kohlenwasserstoffen aus schweren durch kontinuierliche Destillation unter einem Druck von 20-50 Atm. haben E. Gr a ef e und R. v. W a $1 \mathrm{t}$ h e r (D. R. P. 303 883) ausge. arbeitet. Durch Anwendung großer Gas- und Dampfräume bleiben die erzeugten Gase und Dämpfe unter hohem Druck lange miteinander in Berührung, so da $\beta$ der bei der Aufspaltung gebildete Wasserstoff sich an die ungesättigten Kohlenwasserstoffe anlagern kann. - Über Benzol als Motorenbrennstoff, namentlich in Mischung mit Alkohol und Benzin, haben F. A n c k e r (Z. f. Spiritus-Ind. 4\%, 105) und H. B r a c h (Olmotor 8,65-69) geschrieben. Zur Entwässerung von Benzol für Kraftwagenbetrieb empfiehlt A d a m (Autotechnik 8 Heft 4, 2-4) Filtrieren durch einen Trichter mit Hirschledereinlagen. - Die Bestimmung von Benzol in Rohbenzol führt W. J. J o n e s (J. Soc. Chem. Ind. 38, T. 128-130) so durch, daß er das Rohbenzol mit so viel Reinbenzol versetzt, daß der Gefrierpunkt auf $0-1^{\circ}$ steigt, wo er dann dem Gehalt an Benzol proportional ist. Aus den Gewichten und den Gefrierpunkten wird der Gehalt an Benzol berechnet. F. B. J o n e s (J. Soc. Chem. Ind. 37, T. 324-327) gibt eine Anleitung zur Bestimmung von Schwefelkohlenstoff, Thiophen, Toluol und Paraffin in reinen Handelsbenzolen mit Hilfe der Gefrierpunktsemiedrigung gegenüber reinem Benzol, die bei Gegenwart von höchstens $4 \%$ dieser Stoffe nahezu proportional den Gewichtspro. zenten der gelösten Stoffe ist.

$\mathrm{S}$ te in k oh le n t e er. Finen Utberblick über die Bestandteile des Steinkohlenteers und die daraus gewinnbaren Substanzen unter besondorer Perücksichtigung der deutschen Patentliteratur gibt P. E. S pi e $1 \mathrm{~m}$ a $\mathrm{n} n$ (Chem. Trade Journ. 6\%, 471--472 ff.). Auch No e I t i n g (Chimie et Industrie 1, $6 ; 6-70$ ) beschäftigt sich mit dicsem Gogenstand. - Um möglichst wasserfreien Teer zu gewinnen, führen Lo $u$ is $\mathrm{Sc} \mathrm{h}$ war z \& Co. (D. R. P. 312 776)das heiße Teer-Wassergemisch einer Rückkühlanlage (Gradierwerk oder Kaminkühler) zu, in welcher durch Verdunstung und Abkühlung der Mischung in Beriihrung mit bewegter Luft die Entziehung des Wassers erfolgt. Ref. verspricht sich von diesem Verfahren wenig, da das Wasser besondors dort, wo es so innig mit den Teerteilchen vermischt ist wie im Generatorteer, durch Verdunstung nicht abgeht. - Nach einem Verfahren von E. W i $\mathrm{r}$ th (D. R. P. 303 273) warden bei der Reinigung des Teers hochmolekulare harzartige Basen gewonnen und zwar so, daß der Teer nach Entziehung der Phenole zuerst mit stark verdünnter und dann erst mit mäßig konzentrierter Säure ge waschen wird. Dieses Verfahren wird nach D. R. P. 304306 auch auf schwere Teeröle angewendet. - Nach Mitteilung von E. Glas e r (Seife 4, 109-110) wird das Säureharz zur Gewinnung von Cumaronharz benutzt. - W. S c h $\mathrm{midt}$ und $\mathrm{E}$. H e u s ex (D. R. P. 316258 ) laugen den Teer mit Wasser aus und unterwerfen den zu"ückbleibenden Teer der Verseifung. - Uber die Eigenschaften der aus dem Steinkohlenteer hergestellten Tcertettöle gikt K. B r u h n (Stahl u. Eisen 39, 402-406, 469-474) eine zusammenfassende Darstellung. - Teerfettöl eignet sich allein wegen seines Anthracengehaltes und seiner geringen Viscosität schlecht zu Schmierzwecken. Durch Mischung mit Mineralöl verschwinden diese Nachteile (Feuerungstechnik $\boldsymbol{\gamma}, 104-105$ ). Zur Verhütung von Erkrankungen beim Arbeiten mit den Teerfettölen sind, wie P. M. G r e $\mathrm{m}$ p e betont (Braunkohle 13, 164-165), die vom Reichsgesundheitsamt vorgeschlagenen Maßnahmen möglichst zu befolgen, als da sind: besondere Arbeitskleidung, möglichste Vermeidung des Beschmutzens der Hände mit den Schmiermitteln, Einfetten der Haut usw. - Zur Erböhung der Aufnahmefähigkeit von Teerölen für kolloidale Schmierstoffe, wie Graphit, schlägt W 3.0 s tw a ld (D. R. P. 312376 ) Zusatz alkalischer Stoffe, besonders von Magnesiumhydroxyd zwecks Neutralisation der Öle vor. - Nach einem Verfahren der Rö ch I ings chen Eisen-und Stah l werke G. m. b. H. und R. S c hröd er (D. R. P. 315 554) werden Schmier. öle aus dem Teer so hergestellt, daß bis $200-250^{\circ}$ destillierter Teer in heißem Zustande in einem Extrakteur mit Calciumoxyd oder anderen die Harzeäuren bindenden Chemikalien zusammengebracht und nach dem Frkalten mit Alkohol mehrere Stunden gerührt wird. Aus der alkoholischen Öllösung entfallen durch fraktionierte Destil. lation im Vakuum hochgradige Schmierprodukte. - Auf anderer Grundlage beruht das Verfahren von H. K I e v e r (D. R. P. 301 774, 30] 775,301776 und 301 777): unter Druck werden die Teeröle auf $250-350^{\circ}$ erhitzt, gegebenenfalls unter Zusatz von Katalysatoren. Der Druck kann auch durch Luftabschluß oder Gegenwart einer indifferenten Gasatmosphäre ersetzt werden, wobei den kondensierten Dämpfen die Möglichkfit des Rückflusses gegeben ist. Die Verwendung von Rohteer als Dieselmotorenbrennstoff findet immer mehr Verbreitung, so berichtet $R$. E h r lich $\mathrm{E}$ (J. f. Gasbel. 6\%, 37) über einen Dieselmotorbertrieb mit Rohteer in Meiningen, O. S c h e r t l (J. f. Gasbel. 61, 493-495) über Verwendung von Kammerofenteer bei den Dieselmotoren des Wasserwerkes Hamburg. H. M o o $\mathrm{r}$ e (Engineering 108, 167-168) erörtert allgemein die Eignung von Rohteer für diesen Zweck an Stelle von Petroleumdestillaten. - Ein sehr einfaches Verfahren zur Verbesserung von zum Dieselmotorenbetrieb bestimmten Teeröl gibt F. R a s c h i g (D. R. P. 315 030) an: Das Teeröl wird mit geeigneten Mengen Wasser ausgeschüttelt und dann die wässerige Schicht ron dem gereinigten Teeröl getrennt. Dadurch werden die chlorhaltigen Verbindungen beseitigt. - Fine groBe Anzahl von Veröffentlichungen beschäftigt sich mit dem Ersatz der Kohlen. durch die Teerölfeuerung in technischen Betrieben. F. I $\mathrm{m} \mathrm{g} \mathrm{r} \mathrm{u} \mathrm{n} \mathrm{d} \mathrm{(Sprechsaal} \mathrm{52,} \mathrm{70-72)}$ verspricht sich dadurch eine Milderung der Kohlennot, schon deswegen, weil das Teeröl, abgesehen von dem Heizwert von 8000 bis 9000 WE., einen dreimal höheren Nutzeffekt hat im Vergleich zu einer Kohle von mittlerer Qualität. Wcitere Vorzüge wie billigere Kesselbedienung, sauberen Betrieh u. a. führt $R$. H e u B (Z d. Bayr. Revisonsverw. 23, 1) an, ohne sich (Z. d. Bayr. Revisionsverw. 23, 17, 39) den Nachteilen, wie hohem Preis und dergleichen zu vorschließen. - A. S c h m i d (Tonind.-Ztg. 43, 308--310) beschreibt einigo Teerölfeuerungen für Flammenrohrkessel und Drehrohröfen der Firmen Fe 1 l n e r und $\mathrm{Z}$ i e g ler, Frankfurt a. M. - $\mathrm{M}$ a $\mathbf{i}$. $\mathrm{kr}$ a $\mathrm{nz}$ (Wochenschr. f. Brauerei 36, 211-213) weist auf die Gefährlichkeit des Teerölbetriebes hin. Die Gefahr besteht in erster Linie in der Fntstehung zerknallbarer Gase, die bei Verlöschen oder Abreißen der Flamme stattfinden kann (Z. f. Damofkr. Betr. 42, 341-342). Einen solchen Fall behandelt R. H e a B (Allgem. Z. f. Bierbrauerei 47, 235-237), bei dem das Auslöschen der Heizflamme durch schlechten Zug verursacht wurde. - A. F is oher (J.f. Gasbel. 6., 510-513) erstattet Bericht über die während des Krieges entstandene Industrie der Pechverkokung zum Zwecke der Horstellung von Elektrodenkohlen. - Ein anderes Verwertungsverfahren von Pech ist das von W. Her r m a n (D. R. P. 314 996): Der bei hoher Temperatur verflüssigte Pech wird mittels Düsen in Retorten eingestäubt, die von heißen Gasen oder überhitztem Wasser. dampf durchströmt werden. Die Pech+röpfchen geben bereits im Fluge Gase und Dämpfe ab und treffen schon in festem Zustande auf die mit Koks bedeckten Retortenwände auf, so daß ein lockerer Koks entsteht, der sich leicht entfernen läßt. - Über technische Asphalt- und Pechpräparate, ihre Herstellung und ihre Eigenschaften macht E. J. F i s c h e r (Kunststoffe 10, 1-3 ff.) Mitteilungen, die er durch eine tabellarische Übersicht der Patentliteratur ergänzt. Die als ,Teerkrankheit" bekannte, unter Arbeitern von Brikett. 
fabriken auftretende Hautkrankheit wird von Ad. Baget und A. S l o s s e (Compt. rend. 169, 704-706) als Arsenvergiftung an gesprochen. - Eine sehnelle Methode zur Wasserbestimmung im Teer gibt P. S m j tChem. Weekblad 16, 852-854) an: es ist eine Destillationsmethode, nur daß von der angewandten Menge ein Teil im ganzen erhitzt wird, während der Rest in kleinen Mengen so zugegeben wird, daß die Temperatur im Destillationsgefä $\beta$ nicht unter $100^{\circ}$ fällt. - Auch W. S n a 1 t e h o l z (Chem. Weekblad 15, 1546 bis 1548) beschreibt eine Wasserbestimmungsmethode. - H. J. Wa term a n und F. H. C. B a r k h u ysen (Chem. Weekblad 16 , 1251-1257) üben Kritik an dem Kohlenstoffbestimmungsverfahren im Teer, wie es die niederländische Reichskohlenverteilung vorschreibt, und geben die Ergebnisse anderer Methoden an, die sich nach ihrer Ansioht besser eignen. - J. M. W o is s (J. Ind. Eng. Chem. 10, 817-823, 911-916, 1006-1012) teilt eine Reihe von Untersuchungsverfahren mit, die in der amerikanischen Kohlenteerindustrie üblich sind. - H. G. C o $1 \mathrm{~m}$ a $\mathrm{n}$ und E. W. Y e o m a $\mathrm{n}$ (J. Soc. Chem. Ind. 38, T. 57-68, 152) besprechen die Grundlagen und die Ausführung der Benzol-, Toluol- usw. Bestimmung im Kohlenteer und in den Zwischenprodukten der Teerdestillation und teilen ihre diesbezüglichen Erfahrungen mit. - Die Bedeutung physikalischer Methoden für die Kohlenteerindustrie erörtert W. J $H$ u f (J. Ind. Eng. Chem. 11, 1016-1019). -

Tief temperaturteer. H. Strache und M. Doleh (Montan. Rundsch. 11, $409 \mathrm{ff}$.) veröffentlichen die Ergebnisse einer ausgedehnten Untersuchung über die Zusammensetzung von Braunkohlenteeren, hauptsächlich von Vorkommen der. ehemaligen österr. ungarischen Monarchie. Die Untersuchung erfolgte nach dem von F. F is c h er angegebenen Verfahren. - W. S c h n e ider hat (Ges. Abh. z. K. d. K. III. 150-163), um eine möglichste Annäherung der böhmischen Braunkohle an die Steinkohle festzustellen, das Bitumen von zwei böhmischen Braunkohlen untersucht; es haben sich aber hierbei nur Ähnlichkeiten mit der deutschen Braunkohle ergeben. - Über den Stand der Schmierölherstellung aus Urteer gibt die Mineralölversorgungsgesellsehaftin ihren Täglichen Ber. über die Petroleumind. 1919, Nr. 26, Kenntnis. Die Allgemeine Gesells chaft für Che mische In d u s t r i e (D. R. P. 310 653) erhält ans der hochsiedenden Fraktion res Urteers ein viscoses Schmieröl, indem die Fraktion nach Befreiung von ihren sauerstoffhaltigen Bestandteilen mit flüssiger schwefliger Säure behandelt, und die dabei entstehende Lösung von dem sich abscheidenden Paraffin getrennt wird. Das Produkt soll eine Viscosität bis zu $10^{\circ} \mathrm{E}$. bei $50^{\circ}$ haben. - F. F i s c h e r und W. G l u u d (Ges. Abh. z. K. d. K. III. 39-45) haben aus der Fraktion $200-300^{\circ}$ eines Steinkohlenurteers ein bei gewöhnlicher Temperatur flïissiges Paraffin abgesondert. - Bei der langsamen Zersetzungsdestillation haben F. Fis e her und W. Sch neider. (Ges. Abh. z. K. d. K. III. 122-149) aus Braunkohlenteer durch Spaltung der hochmolekularen, über $300^{\circ}$ siedenden Anteile auch Benzine mit teilweise unter $100^{\circ}$ siedenden Teilen erhalten. Diese Benzine haben dem Petroleumbenzin gegenüber die Nachteile des scharfen, auch durch Raffination nicht $\mathrm{zu}$ entfernenden Geruches und des hohen Gehaltes an ungesättigten Verbindungen. - Versuche von $W$. G l u u d (Ges. Abh. z. K. d. K. III. 46-65) über die Gewinnung von Leuchtölen, Treibölen und dergleichen aus Steinkohle haben ergeben, daß sich aus den Kohlenwasserstoffen des Steinkohlenurteers die gleichen Produkte herstellen lassen wie aus Braunkohlenschwelteer. - Die Frage, ob sich aus den Bestandteilen des Steinkohlenurteers Cumaronharz gewinnen läBt, beantworten $G l u$ u d und B r e u e r (Ges. Abh. z. K. d. K. III. 238-242) auf Grund ihrer Versuche dahin, daß sich aus den Kohlenwasserstoffen der Fraktion $155-185^{\circ}$ dieses Harz nicht herstellen läBt, daß jedoch die Phenole des Urteers bei Krackdestillation und Verarbeitung der in Frage kommenden Fraktion Cumaronharz ergeben. Daraus wird geschlossen, daß die Cumaronharze ihre Bildung in erster Linie der Überhitzung von primär gebildeten Urteerphenolen verdanken. - F. $\mathrm{F}$ is $\mathrm{c}$ ber und GI u a d (Ges. Abh. Z. K. d. K. III. 109-121) haben ferner die Eigenschaften und die.Verwendbarkeit der aus Urteer durch einfache Destillation ohne Entfernung der Phenolo erhaltenen Produkte festgestellt. - Was die Unterschiede des Braunkohlenurteers von gewôhnlichem Schwelteer anlangt, so sind dieselben nicht so groß, wie die Unterschiede zwischen Steinkohlenurteer und gewöhnlichem Steinkohlenteer. Sie sind mehr quantitativer Natur, wie F. F i s c her und W. Schneider (Ges. Abh. z. K. d. K. III. 200-212) gefunden haben. Zur raschen Orientierung dient ein Verfahren, das die quantitative Bestimmung des in Petroleum äther Unlöslichen gestattet: Braunkohlenurteere ergeben einen höheren Prozentgehalt an Unlöslichem (über 30\%) als andere Braun kohlenteere (unter 10\%). Von Steinkohlenurteer unterscheidet sich Braunkohlenurteer durch seine Konsistenz und durch seinen Methoxylgehalt. - E. E r d m a n n (Braunkohle 17, 425-428, 435-437) macht den Vorschlag, Braunkohlenteer mit Hilfe von Aceton auf zuarbeiten, eine Methode, die vorher aber auch schon von F. Fi scher, W. Gluud, W. Schneider und H. Tropsch angewendet worden ist. - Eine andere, vielversprechende Verwertungsart von Braunkohlenteer ist die Oxydation der Braunkohlen. teeröle zu Fettsäuren und Verarbeitung der lezteren zu Seifen. Es kommen hier vor allem die Patente von C. Harries, E. A l b r e c h t und R. K o e t s c h a u (D. R. P. $314745,314746,314747$ ) in Betracht, ferner die Versuche von $\mathrm{B}$ e $\mathrm{r} \mathrm{g}$ m a $\mathrm{n} \mathbf{n}$ (Angew. Chem. 31, I, 69), der Paraffin mit Luft zu Fettsäuren oxydiert hat

$\mathrm{N}$ a p h t ha I i n. Aus hydrierten Naphthalinen oder Naphthalinabkömmlingen werden durch Polymerisation Verbindungen er halten die als Schmierölersatz dienen können. Das Verfahren zur Herstellung dieser Körper ist der T e t r a l i n G. m. b. H. (D. R. P. 309 178) geschützt. - Die Verwertung von Naphthalin zur Energie erzeugung in Naphthalinmotoren ist schon bekannt. Eine von F. S chröde r (D. R. P. 312 189) konstrujerte Maschine benutzt als Wärmeträger des zum Schmelzen des Naphthalins dienenden Wärmestromkreises eine Flüssigkeit mit einem Siedepunkt über $100^{\circ}$ Dieser Wärmestromkreis wird als Wärmequelle für den zum An wärmen der Maschine dienenden Wasser als Wärmeträger führenden Wärmestromkreis verwendet.

Gasreinigungs masse.A. Hutin (Rev. de chimie ind 27, 147-150) beschreibt drei Methoden zur Verarbeitung von Gasreinigungsmasse: 1. Gewinnung von Schwefel, Ammoniak, Rhodansalzen und Ferrocyaniden, 2. Gewinnung von unreinem Schwefe neben den Salzen wie in 1, und 3. Wiedergewinnung des Schwefels als $\mathrm{SO}_{2}$ oder in Form von Polysulfiden. - Da die Bestimmung des Schwefels in ausgebrauchter Masse mit Schwefflkohlenstoff zu hohe Resultate gibt, so schlägt W. D i a m o n d (J. Soc. Chem. Ind. 3r, T. 336-337) eine Vorbehandlung mit Benzol vor. Die Benzolaus züge müssen aber, da sie Teile von Schwefel enthalten, zur Bestimmung desselben oxydiert, una der $\mathrm{S}$ als $\mathrm{BaSO}_{4}$ gewogen werden. $\mathrm{T} w$ is s e l man $\mathrm{n}$ (Chem.-Ztg. 42, 558) bestimmt den Reinschwefel, indem er aus einem Teil des Schwefelkohlenstoffextraktes den Schwefel durch Erhitzen sublimiert und den Rückstand wägt. Fine Verbesserung des Drehs chmidt schen Extraktionsauf satzes zur Schwefelextraktion beschreibt $K$. Fu n ck (Cher. Ztg. 42, 534).

[A. 112.]

\section{Über Essigsäureabspaltung aus Holz und Lignin.}

(Erwiderung auf die Mitteilungen von Hans Pringsheim und Hans Magnus ${ }^{1}$ ).

Von Carr G. Schwalbe und Ernst Beckmi.

(Mitteflungen aus der Versuchsstation für Zellstoff- und Holzchemie in Eberswalde.)

(Eingeg. 5./8. 1920.)

In der in der Überschrift erwähnten Mitteilung von $\mathbf{P}$ r in $\mathbf{g} \mathbf{s}$. $\mathrm{h}$ e $\mathrm{i} \mathrm{m}$ und $\mathrm{M}$ a $\mathrm{g} \mathrm{n}$ u beanstandeten die Verfasser vor allem, daB wir angeblich ihre Arbeit nach einem Zentralblattreferat kritisiert hätten. Dieser Vorwurf trifft aber keineswegs zu. Unsere Kritik bezog sich auf die mit einem Vorwort von $\mathrm{P}$ r i $\mathrm{n} \mathrm{g} \mathrm{s} \mathrm{h} \mathrm{e} \mathrm{i} \mathrm{m} \mathrm{versehene}$ Sonderschrift von $\mathrm{M}$ a g n u s: ,Theorie und Praxis der Strohaufschließung", nicht aber auf ein Zentralblattreferat, das uns erst während der Korrektur bekannt wurde. Unsere kritischen Aus

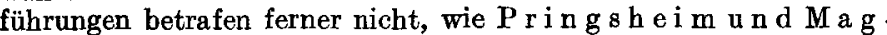
n u s ansfithren, den Vergleich zwischen WeiBbuchenholz- und Nadel holzlignin, sondern denjenigen zwischen Kiefer- und Strohlignin, ein Vergleich, der in der erwähnten Schrift von $M$ a g $\mathbf{n}$ u s gezogen worden ist.

Unsere von $\mathbf{P}$. und $\mathbf{M}$. beanstandete $\mathrm{K}$ ritik richtet sich gegen die

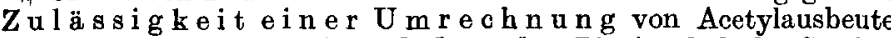
auf Ligningehalt. Wenn $\mathrm{P}$. und $\mathrm{M}$. aus dem Ligningehalt des Strohes mit 23\% und der zugehörigen Essigsäureausbeute von 4,9\% folgern daß man bei Kiefer mit 30\% Ligningehalt 5,7\% Essigsüure zu erwarten habe, so behaupten sie doch damit, daß man aus dem Ligningehalt auf Essigsäureausbeute schließen darf. Wir aber fanden, daß bei viel vorhandenem Lignin die Essigsäureausbeute, z. B im Fichten- und Kiefernholz, weit geringer sein kann, als bei einem weniger Lignin enthaltenden Laubholz, wie Birke, Buche und Pappel, ein Befund, der die uns damals noch nicht bekannten Ergebnisse in der $z$ we i te $n$ Arbeit von $P$. und $M$. bestätigt.

Von dieser von uns bekämpften Anschauung der Zulässigkeit einer Umrechnung sind $\mathrm{P}$. und $\mathrm{M}$. in der neueren Abhandlung in der Z. f. physiol. Chem. - die uns unter den gegen wärtigen schwierigen Zeitverhältnissen erst jetzt im Original zugänglich geworden ist offenbar völlig abgekommen. Auch P. und M. sind jetzt der Meinung, daß niederer Ligningehalt mit hoher Essigsäureausbeute und umgekehrt hoher Ligningehalt und niedere Essigsäureausbeute, parallel gehen können.

Im übrigen können wir uns der Beweisführung von $P$. und $M$. in ihrer Erwiderung ${ }^{2}$, daß unsere nach der Methode von $\mathrm{S}$ c h o r g e $r$ ermittelten Werte ,falsch sind, weil die ganze Methode falsch sein muß und ganz einfach nicht die Abspaltung der Gesamtmenge

1) P r ing s h e i m und M a g n u s, Angew. Chem. 33, 56 [1920], Nr. 18.

) Angew. Chem. 33, 56 [1920]. 